\title{
A pilot randomised controlled trial comparing a health-related lifestyle self-management intervention with standard cardiac rehabilitation following an acute cardiac event: implications for a larger clinical trial
}

\begin{abstract}
Purpose This pilot study was to assess the feasibility of the Health-related Lifestyle selfManagement (HeLM) intervention as a strategy to decrease cardiovascular risk following acute coronary syndrome.
\end{abstract}

Methods Participants in this randomised controlled trial were recruited from a tertiary teaching hospital in metropolitan Sydney Australia. The multifaceted HeLM intervention, using the principles of the transtheoretical model, involved the use of bibliotherapy, a structured evidencebased approach to cardiovascular risk reduction, a communication strategy with general practitioners, three supportive telephone calls to participants and provision of behavioural prompts and a health record diary. Differences in behavioural and clinical outcomes between the HeLM intervention group $(n=29)$ and the standard cardiac rehabilitation group $(n=22)$ were assessed. Results A total of 125 participants screened were eligible for participation in the study. Fifty-one participants, mean age 57 years $( \pm 8.78)$ were randomized. At the 8-week follow-up, participants in the HeLM intervention group had a reduced systolic blood pressure compared to the standard care group (120.3 SD: 16.3 vs 126.4 SD:14.6). There were no significant differences in diastolic blood pressure and cholesterol levels between the two groups. Participants in both the intervention and control group had a reduction in waist circumference although when compared to baseline values, women in the HeLM intervention group had a greater reduction compared to those receiving standard care. Patients reported high levels of satisfaction with this intervention.

Conclusions: Findings support the feasibility of implementing the Health-related Lifestyle selfmanagement intervention for risk factor modification in patients with acute coronary syndrome. An 
adequately powered randomised controlled trial is required to test the impact of the intervention on cardiovascular risk reduction.

Key words: secondary prevention, brief intervention, acute coronary syndrome, transtheoretical model, feasibility study

Funding: Funding for this pilot study was obtained from the Health Research Foundation Sydney South West, Sydney Australia. 


\section{Burden of coronary heart disease}

Coronary heart disease (CHD) remains the leading cause of mortality and morbidity globally, ${ }^{1,2}$ placing significant burden on both individuals and the community due to disability and associated costs ${ }^{3}$. Lifestyle modification focussing on behavioural changes to address modifiable risk including smoking, ${ }^{4},{ }^{5}$ physical inactivity, ${ }^{4}$ hypertension, ${ }^{4}$ dyslipidaemia, ${ }^{4}$ hyperglycemia, ${ }^{4}$ and obesity, ${ }^{4}$ is essential to minimise CHD progression ${ }^{6}$ and to decrease cardiac mortality and morbidity.

Participation in cardiac rehabilitation (CR) has been shown to improve quality of life, reduce mortality by $35 \%$ and reduce cardiovascular risk through secondary prevention strategies. ${ }^{7}$ In spite of these benefits, a number of secondary prevention programs $s^{7-10}$ have been implemented with limited participation rates. Poor participation in these programs has been attributed mainly to limited availability and accessibility, length of the program (ranging from 4 weeks to 1 year), distance from home or work, time conflicts, lack of physician/ family support and transportation and scheduling of classes. ${ }^{11-13}$

Innovative methods such as individualized coaching ${ }^{8}$ and modular approaches ${ }^{9}$ have been implemented for the delivery of information relating to cardiac risk factors for patients with CHD. However, none of these approaches assess the patients for their readiness to change and therefore the tailoring of strategies to individual needs remain questionable.

Brief interventions (BI) are another strategy that is widely used for risk factor modification. A BI is a tool consisting of time-limited, structured advice focusing on changing and/or increasing desirable patient behaviour. ${ }^{14}$ The main aim of a BI is to leverage individual's motivation to change, following which they may continue to change their behaviours with minimal formal intervention 
and thereby reduce their risk factors for further CHD. Evidence obtained from a systematic review of randomised controlled trials (RCTs) ${ }^{15}$ demonstrated a statistically significant decrease in the incidence of smoking, mean fat intake and body mass index and a significant increase in participation in physical activity at 6 month follow-up, among CHD patients randomised to a structured BI compared to those who received standard care. Based on these findings a health related lifestyle self-management (HeLM) that was structured and designed to be brief was developed as an alternate cardiovascular risk factor reduction strategy.

This pilot randomised controlled trial was undertaken to compare the effects of the HeLM intervention with standard care on behavioural and clinical changes following an acute cardiac event. Information was also sought relating to participant recruitment, study procedures and compliance to treatment.

\section{The HeLM intervention}

The HeLM intervention is a structured brief cognitive behavioural intervention program based on the transtheoretical (TTM) model of behaviour change. It comprised of goal-setting, bibliotherapy, feedback of personal risk, collaboration and communication with GPs, motivational telephone calls, a refrigerator magnet and a health diary.

Goal-setting Goal-setting was undertaken according to the SMART principles. ${ }^{16}$ The acronym SMART stands for Specific, Measurable, Achievable, Realistic and Time. Patients were asked to write down specific and realistic goals they would like to achieve.

Bibliotherapy A distinct feature of the HeLM intervention was the use bibliotherapy in the form of a 50 page colour-coded booklet titled Take the HeLM. This booklet was developed as a self-help resource to provide skills training for modification of health risk-related behaviour relating to smoking, physical activity and saturated fat intake. The 50-page booklet was designed to have a 
colour-coded section corresponding to each of the five stages of change for each of the health riskrelated behaviours. Specific strategies that the patients could activate in order to progress to the next stage were listed in each stage of change section. This method ensured that the information in the booklet was tailored to the individual. ${ }^{17}$ The booklet included the meaning of and the risk factors for CHD and participants were asked to identify the risk factors that applied to them. It also included a short quiz that enabled the patients to identify their stage of change and a section that provided information and strategies corresponding to the stage of change for each of the health risk factors. Extensive measures including calculating the Flesch Reading Ease score ${ }^{18}$ and the Flesch-Kincaid Grade Level score to ensure comprehensibility of the HeLM Booklet were undertaken. The reading level of the HeLM booklet was targeted at a Year Six stage as findings from a previous study demonstrated that the majority of patients had left school before secondary education and $47.8 \%$ had neither completed secondary school nor attained any subsequent qualifications. ${ }^{19}$ The HeLM booklet was subsequently given to a Year Six student and three patients for assessment of readability.

\section{Feedback of personal risk Risk factor card}

Based on the patient's physical and laboratory data individual risk factor cards were generated that included the values for all their cardiovascular risk factors along with the normal values for comparison. All abnormal values were highlighted. A five year mortality risk score and the risk of developing CHD or having a recurrent CHD event was calculated and presented from the results obtained using a cardio risk calculator provided by Merck, Sharpe \& Dohme. The risk factor calculator is based on the Framingham Heart Study ${ }^{20}$ in the USA.

\section{Personalised letter}

Participants were mailed a personalised letter providing feedback about their existing habits relating to smoking, exercise and diet and how they felt about modifying these risk-related behaviours. This 
feedback enabled them to read the appropriate section of the HeLM booklet for strategies for risk modification.

\section{Collaboration and communication with general practitioners}

The general practitioners (GPs) of participants in both groups received a copy of the Reducing Risk in Heart Disease (RRiHD) ${ }^{6}$ guidelines, participant's risk factor status, and the goal that the participant has agreed to achieve. GPs were required to provide support for these patients and reinforce lifestyle changes and inform participants of the exact values of their biomedical parameters to empower them.

Three supportive telephone calls The HeLM intervention included three supportive phone calls during the 8-week intervention period. The phones calls were delivered by a research assistant, with previous experience and training in motivational interviewing. These phone calls were conducted at weeks two, four and five following discharge from the hospital and each call was limited to 15-20 minutes These phone calls strictly focussed on providing motivational support and encouragement and to monitor progress.

Fridge magnet Participants were given a fridge magnet that provided information on what to do in an emergency.

Health diary for self-monitoring Participants were given a health diary where they could selfmonitor their results, e.g. blood pressure, cholesterol, weight, and physical activity, with room to document subsequent values.

The HeLM intervention was conducted over a 6- week period. Most secondary prevention interventions are conducted face to face and include provision of information in an unstructured manner. The HeLM intervention is novel as it incorporates the TTM of behaviour change within a $\mathrm{BI}$ and is conducted in a less intensive format than conventional CR programs using telephone and postal communication. The HeLM intervention is designed to provide self management strategies, 
and brief motivational support using the telephone at a time suitable to patients. The HeLM intervention will potentially overcome barriers, such as waiting lists, transportation and scheduling of classes associated with participation in traditional CR. ${ }^{11-13}$ The information strategies provided in the HeLM intervention can be accessed by participants in their own time. Thus the HeLM intervention is potentially useful for individuals in regional and remote areas, those without transport or who are working, particularly those working shift work.

\section{Standard care}

The control group received standard care, which included providing information relating to heart disease by health professionals in the clinical area.

As part of the study protocol participants in both groups were given the name and contact details of the CR centre closest to their home.

\section{Methods}

\section{Participants}

Study participants were recruited from the cardiology wards of a metropolitan tertiary teaching hospital in Sydney Australia between July 29-October 10 2006. Patients were included if they met the following eligibility criteria: aged 18 years or older; admitted to hospital with a diagnosis of an ACS, ${ }^{21}$ provided written consent and had one or more of the following modifiable risk factors:

a) smoking (more than 1 cigarette per day ${ }^{6}$ or a breath Carbon monoxide (CO) level of 6 or 8 $\left.\mathrm{ppm}^{22}\right)$

b)overweight and obesity as determined by body mass index (BMI) $>25 \mathrm{~kg} / \mathrm{m}^{26}$ and increased waist measurement ( $\leq 94 \mathrm{~cm}$ males and $\leq 80 \mathrm{~cm}$ for females); ${ }^{23}$

(c) high saturated fat intake (more than 21 points) as measured by the 17-item Short Fat questionnaire; ${ }^{24}$ 
(d) low physical activity (less than 30 minutes of moderate intensity physical activity per day for most days of the week (150 minutes per week minimum) ${ }^{6}$;

(e) documented history of high total cholesterol $(>4 \mathrm{mmol} / \mathrm{L})^{6}$; and

(f) documented history of hypertension ( $\mathrm{BP}>140 / 90 \mathrm{~mm}$ Hg if the patient is $\geq .65$ years; $\mathrm{BP}>130$ mmHg if the patient is $\leq 130 / 85 \mathrm{~mm} \mathrm{Hg}$ and $\mathrm{BP}>125 / 75 \mathrm{~mm} \mathrm{Hg}$ if the patient has proteinuria. ${ }^{6}$

Patients were excluded if: (1) they had major comorbidities, such as stroke and cancer that would complicate their convalescence; (2) their physician did not want them to participate in the trial; (3) they were unavailable for long-term follow-up; or (4) they were unable to understand English and personally give informed consent.

\section{Recruitment strategies}

The cardiologists, CR coordinators and the nursing unit manager in the participating hospital were provided with details of the project. Clinicians in the participating wards were briefed about study, however, no details of the intervention were given to avoid contamination of the intervention. The nursing unit manager was requested to nominate a registered nurse who had knowledge of the patients' diagnoses and treatments to act as a liaison between research personnel and the ward staff. To identify patients, the liaison person was given a copy of the inclusion/exclusion criteria for the study. Recruitment to the study was undertaken by a research assistant who met the liaison person to identify potential participants. The clinical notes of these patients were reviewed to confirm eligibility. Patients suitable for the study were given a brief overview of the study along with a subject information sheet, and written consent was obtained.

\section{Randomisation}


Randomisation was performed by a statistician who was blinded to the trial details and who had no connection with the study. After recruiting each patient, the research assistant gave the statistician the patient's medical record number and continued to collect baseline data with no knowledge of the patient's group allocation. The statistician randomly assigned the patients to either the HeLM intervention or standard care using a random numbers table ${ }^{25}$. Allocation concealment was undertaken using sealed opaque envelopes.

Participants in both groups were contacted at week six of the intervention to organise a date and time for the 8-week clinic visit for data collection. Three attempts were made to contact the participants, after which they were declared lost to follow-up. The same data collection instrument was used for both the baseline and 6-week follow-up.

All patients who attended the follow-up visit were given a thank-you letter, pens, a recipe book from the National Heart Foundation of Australia, a pedometer, and a copy of the RRiHD guidelines. $^{26}$

\section{Outcome Assessment}

Data were collected relating to clinical and behavioural risk factors, participation in Phase 2 cardiac rehabilitation and the feasibility of the study.

\section{Clinical risk factors}

The clinical risk factors investigated were incidence of Major Acute Coronary Event (MACE) including death and readmission to hospital for an acute coronary event, serum lipid level and blood pressure level. Baseline serum lipid levels were obtained from clinical records. Self-reports were used to identify incidence of MACE and follow-up serum lipid levels. The last blood pressure measurement that was recorded in the medical charts was used for baseline data. For the follow-up data collection, the blood pressure was manually measured according to the American Heart 
Association guidelines ${ }^{27}$ using a sphygmomanometer and stethoscope. Recommendations from the RRiHD $^{26}$ were used as indicators for all outcomes .

\section{Behavioural risk factors}

Behavioural risk factors assessed at baseline and follow-up were smoking status, saturated fat, fruit and vegetable intake and participation in physical activity.

Smoking status was assessed using self-reports ${ }^{28}$ and carbon monoxide (CO) monitoring. A breath CO level of 6 or 8 ppm $^{22}$ was taken as the cut-off between smokers and non-smokers. Dietary saturated fat intake was determined by using a 17 -item validated Short Fat questionnaire. ${ }^{24}$ Dietary fruit and vegetable intake was assessed using a 2-item validated fruit and vegetable intake questionnaire. ${ }^{29}$ One serve of fruit was considered to be 150 grams of fresh fruit and one serve of vegetables was 75 grams. ${ }^{30}$ Exercise habits were assessed using the Active Australia survey ${ }^{31}$ which reports on the frequency and duration of a range of activities performed within a broad class of physical activity intensity. ${ }^{31}$ Anthropometric measurements including height, weight, and waist circumference were measured to assess obesity. Information relating to participation in cardiac rehabilitation was obtained through participants' self reports and was not verified with source data.

\section{Feasibility outcomes}

The feasibility outcomes investigated were patient recruitment, protocol logistics, and acceptability of the intervention. Protocol logistics was determined by assessing the suitability of data collection methods and the completion of the questionnaires by participants. Acceptability was assessed by asking the participants to rate their satisfaction with the different components of the HeLM intervention on a Likert scale that ranged from 0 (poor) to 10 (excellent). In addition, participants were asked to provide personal comments about the intervention. 


\section{Statistical analysis}

All responses received were coded to ensure confidentiality. The data were collated by the RA who was not blinded to the group allocation and analysed using SPSS 13.0. Statistical analyses were based on intent to treat and significance level was set at $p<.05$. Changes from baseline to 6-week after the intervention for behavioural and clinical outcomes was performed by using a two-tailed and independent Student $t$, and [chi] ${ }^{2}$ tests, as appropriate. Recommendations from the RRiHD guidelines ${ }^{6}$ were used for all outcomes. Approval to conduct the study was obtained from a health service and university. .

\section{Results}

\section{Participant Characteristics}

A total of 547 patients were discharged from the participating wards during the study period of whom 125 were eligible for the study. Fifty one patients agreed to participate and the remaining refused for various reasons (Figure 1). There were no statistically significant differences between the dropouts and the responders on gender, employment status and marital status. However, responders were significantly older $(p=0.005)$ and had more years of schooling $(p=0.040)$ compared to the non-responders. Seventy-eight percent $(n=40)$ of the participants in the study were male and $22 \%(n=11)$ were female. The mean age of the participants was 57 years $( \pm 8.78 \mathrm{SD})$. There were no significant differences between the intervention and control groups in demographic characteristics at baseline. (Table 1)

\section{Clinical outcomes}

\section{Incidence of Major Coronary Events (MACE)}

None of the patients who were contacted for the 6-week follow-up had died, required rehospitalisation for myocardial infarction or an unplanned revascularisation, or had a cardiac arrest. 
Three participants were readmitted to hospital, of whom, one was admitted for non-cardiac reasons and the remaining two for revascularisation of the coronary arteries that could not be performed at the initial admission.

At baseline there was no statistically significant difference between the HeLM intervention group and the standard care groups relating to blood pressure, serum cholesterol level, BMI and waist circumference of patients. At the 8-week follow-up, compared to baseline values, patients in the intervention group had increased systolic blood pressure while those in the control group had a reduced systolic blood pressure. However, the difference in the systolic blood pressure from baseline to follow-up between the two groups was not statistically significant. There was a nonsignificant increase in diastolic blood pressure in both groups from baseline to follow-up. (Table 2) Cholesterol results were available for only 20 and 13 patients in the intervention group at baseline and follow-up and from 17 and 11 patients in the standard care group at baseline and follow-up. The mean cholesterol level in the intervention group reduced from $4.6 \pm 1.5$ at baseline to $4.1 \pm 1.3$ at follow-up. Similar differences were noted in the control group At baseline and follow-up, the mean waist circumference for both males and females was higher in both groups compared to the recommendations in the RRiHD guidelines. The mean waist circumference reduced by $1.1 \mathrm{~cm}$ in males randomised to the HeLM intervention and by $1.4 \mathrm{~cm}$ in those who received standard care. However in females, there was a statistically non significant reduction in $4.4 \mathrm{~cm}$ and $1.3 \mathrm{~cm}$ in waist circumference among those randomised to the HeLM intervention and standard care respectively. (Table 2)

The mean BMI in both groups was higher than the recommendations from the RRiHD guidelines. There was a slight reduction in the mean BMI in both groups from baseline to follow-up. (Table 2)

\section{Behavioural outcomes}


At baseline there was no statistically significant difference between the intervention and control groups in the number of participants who smoked, and at the 8-week follow-up, one participant in each group stopped smoking. The mean carbon monoxide level in both groups was 1.8ppm and there was no difference in this level between baseline and follow-up. Participants in the intervention group reported to be walking for a mean of 98 minutes each week at baseline which increased to 237 minutes/week at follow-up ( $p=0.18)$. In the control group however there was no difference in the total time walked between baseline and follow-up. A greater number of participants $(n=14)$ in the intervention group reported to be engaging in 150 minutes of physical activity each week $(n=14)$ at follow-up

The mean fat intake for participants in the standard care group at baseline was $18.8 \pm 6.8$ than those in the HeLM intervention group (Mean 16.5 \pm 6.2 ). However, at the 8-week follow-up, participants in both groups had a non significant reduction in fat intake.

Participants in both groups consumed the number of servings of fruit according to the recommendations from the RRiHD guidelines. However, at the 8-week follow-up participants in the standard care group had a reduction and those in the intervention group had an increase in the number of servings of fruit per day $(p=0.03)$.

Unlike fruit intake, vegetable intake was poor among participants in both groups at baseline. At the 8-week follow-up, however, participants in the HeLM intervention group increased their vegetable intake unlike those in the control group. These increases in vegetable intake were also below the recommendations from the RRiHD guidelines.

\section{Participation in cardiac rehabilitation}


Only three participants randomised to the HeLM intervention and one to the control group participated in Phase 2 CR. When asked if they were contacted by the CR nurse, 28 participants indicated that they were not contacted by any health professional other than the RA. One of the 28 participants who was not contacted by the CR coordinator but participated in CR reported that he attended following advice from the RA.

\section{Feasibility Outcomes}

Patient recruitment, including review of the exclusion criteria checklist, took an average of 5 minutes. The total time required of the patient was one hour. The personnel required to complete the HeLM intervention was a health professional experienced in motivational interviewing supervised by a registered nurse with a $\mathrm{PhD}$. One research centre staff was needed to randomise the patients to study groups and a liaison staff to identify patients in the wards. Finally, patients needed an easily accessible place that was also private place to complete the questionnaires.

Blood pressure readings were available for all participants. However, none of the patients recruited to the study had their serum lipid level measured. Data for these clinical outcomes were obtained from self-reports of patients who could remember the values. At baseline assessment the CO level registered for the participants who smoked was consistently false negative. This result could be due to the timing of the assessment of CO levels rather than the Smokelyser, as many of these patients had not smoked for at least 24 hours prior to participating in the study, thus allowing for CO dissipation. $^{32,33}$ Therefore, the usefulness of the assessment of the CO level in this study at baseline remains questionable.

Most of the participants completed the entire questionnaire without assistance, however five required assistance with some sections and three preferred the questionnaire to be read to them. A 
small proportion of participants for whom English was a second language had difficulties understanding some terminology used in the validated scales within the questionnaire, and required assistance from the researcher for completing the questionnaire.

Overall participants who received the HeLM intervention reported it to be extremely useful. Ninety percent of the patients stated that the timing for receiving the intervention was appropriate. All participants reported that the information provided in the Take the HeLM booklet was easy to read, could be understood easily and was useful. They also indicated that after reading the booklet they were able to see the benefits of adopting healthy habits, increase their confidence in being healthy and learnt to cope with difficult situations. Participants also commented that their partners and other members of their family found the HeLM booklet useful as it encouraged and supported them to adopt healthy lifestyle habits.

Seven participants indicated that they knew of only some of their personal risk factors and two indicated that they were not aware of any of their risk factors prior to receiving the risk factor card. None of them were aware that once having had a cardiac event, the risk of another was approximately 20\%. ${ }^{34}$ Participants indicated that the risk factor card made them think about the health-related behaviour that they could change. Letters were sent to the GPs of all participants in the intervention group. However, none of them made any verbal or written contact with the researchers. It could be inferred that the GPs had no objection to the intervention. In general, no major issues were identified with any specific aspects of the intervention, suggesting that the content of the HeLM intervention was acceptable.

All participants in the intervention group stated that they valued the follow-up phone support and felt free to talk to the interviewer. The length of the telephone call varied between 5 and 30 minutes 
(mean length of time 17 minutes). They indicated that the telephone support motivated them to commence health-related behaviour changes.

Patients reported that the health diary was reported to be extremely to keep track of their clinical results.

\section{Discussion}

This pilot randomised controlled trial was undertaken to determine the feasibility of the HeLM intervention for risk factor modification in patients with ACS. In this study, $78 \%$ of the participants were male and $22 \%$ were female. Although this ratio of male to female participation is unequal, it is consistent with other trials undertaken in the cardiac population. ${ }^{35,36}$ Baseline characteristics in the intervention and control groups were comparable, which demonstrates success of the randomisation

methods. ${ }^{37}$ Participation in physical activity, and mean changes in BMI, WTHR, fat intake, and fruit and vegetable intake were greater in the intervention group. There was no difference in the number of people who had given up smoking between the two groups. The findings from the study are expected as the study was not adequately powered to determine differences in outcomes. Although, conclusions cannot be drawn from these findings it highlights the difference in outcomes between the two groups. It was clear from the participants that the HeLM intervention succeeded in providing them with risk factor education and motivation to change behaviour.

The study methods were found to be feasible despite some limitations which have highlighted areas where the methods could be refined to ensure a larger study could be undertaken more effectively and efficiently. First, approximately half of eligible patients were missed because they were not able to be interviewed before their discharge time of $10 \mathrm{am}$ and they could not be kept due to pressure on hospital beds. Others refused to participate mainly due to inability to return for the follow-up data collection, work commitments and distance. This finding is consistent with reasons for patients not participating in CR programs. ${ }^{11-13}$ Including these patients may have altered our results. The HeLM 
intervention has been designed to target these patients, therefore, future research should look into home visits for data collection to demonstrate the benefit of the HeLM intervention. Secondly limited resources prevented screening for cognitive status in patients older than 75 years who would benefit from the HeLM intervention. The inclusion of a validated instrument to assess cognitive function and ADLs in future trials will increase the proportion of eligible participants. The length of the questionnaire was also a limitation and therefore items identified as redundant should be removed for the larger study. It was not possible to calculate exact risk factor scores for patients using the Framingham calculator because the majority of them did not have cholesterol levels monitored. This could be due to the fact that all patients were receiving HMG-CoA reductase inhibitors, which are standard treatment for ACS, and that cholesterol monitoring in the acute phase would produce inaccurate results. The use of other risk factor calculators which do not rely on cholesterol values for risk calculation should be considered during the design of a larger study. Cholesterol monitoring by the GP at follow-up should be implemented in the larger trial.

As is well-known a large number of participants had returned to work after their cardiac event, and were unable to be contacted to complete the intervention. To overcome the communication challenges, the researcher utilised other strategies such as voice messages with limited success. The use of text messaging and or providing after hours personnel to deliver the intervention should be investigated in future studies. ${ }^{38}$

The HeLM study holds several strengths. Firstly, delivery of the intervention by a single trained person ensured that the intervention was delivered uniformly to all participants in the intervention group. This intervention was developed based on the principles of evidence-based practice and embedded in a theoretical framework namely the TTM that has been demonstrated to be successful in behaviour change. Individuals may have more than one risk factor and could be at different stages for each of these risk factors; the Take the HeLM booklet was therefore designed to 
incorporate the different stages of change for each of the behavioural risk factors. The HeLM intervention is potentially a useful model for secondary prevention as it can be delivered to a large population in a short time and can be accessed remotely. In addition, the HeLM intervention may be able to be adapted to other chronic diseases

\section{Conclusion}

The HeLM intervention has been developed as an option for is an option as a secondary prevention strategy to reduce cardiovascular risk and potentially has some advantages in terms of access and accessibility. We plan to systematically evaluate the hypothesis posed in this preliminary study in a larger trial to assess the effectiveness of the HeLM intervention to improve cardiovascular risk reduction, both in the short and long term. The systematic pilot study underscores the importance of preliminary studies to adequately determine sample size to detect differences between groups, assessing methods for recruitment and retention.

\section{Acknowledgements}

The authors gratefully acknowledge the financial assistance provided by the Health Research Foundation Sydney South West Area Health Service, Australia for the conduct of this study. The authors would also like to thank the staff of the cardiology wards and the patients who participated in this research.

Conflict of Interest: none declared 
Figure 1 Flow of patients through the study

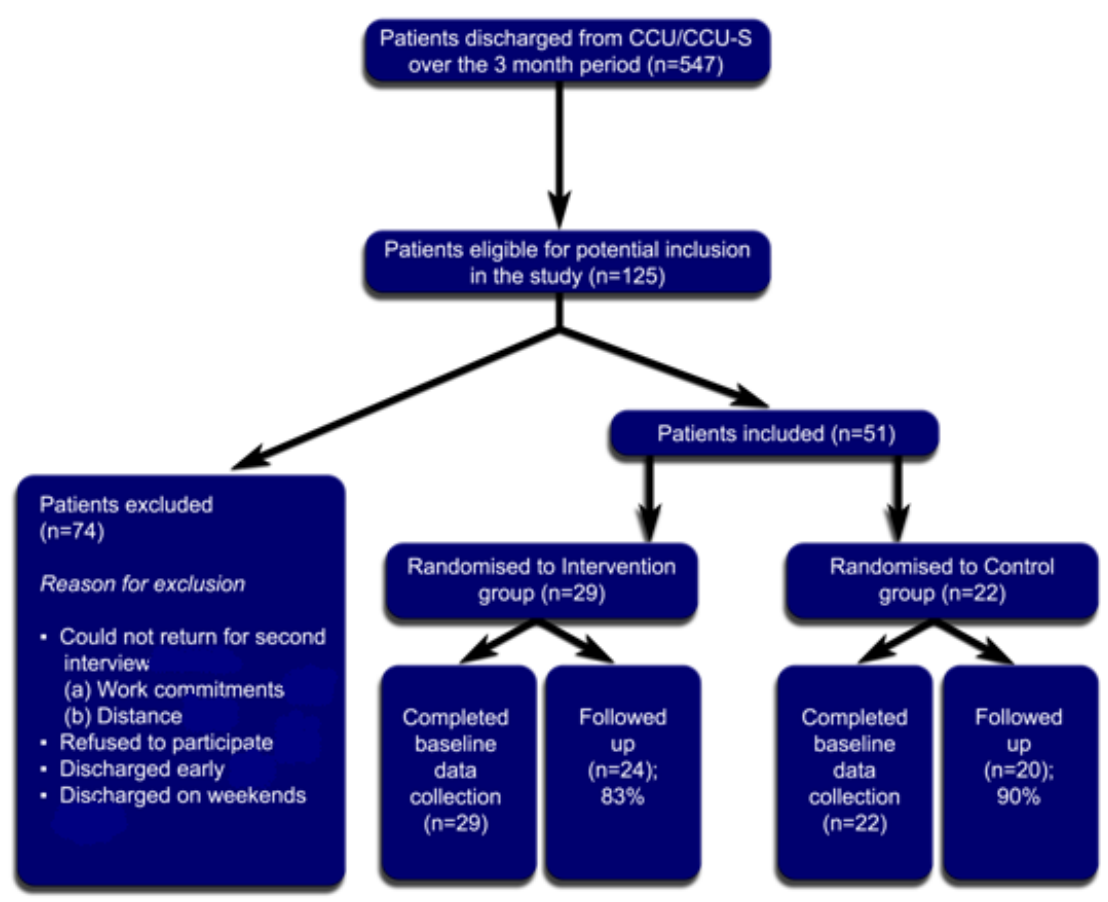


Table 1 Demographic data of the participants

\begin{tabular}{llll}
\hline & $\begin{array}{l}\text { Intervention } \\
(\mathbf{n = 2 9 )}\end{array}$ & $\begin{array}{l}\text { Control } \\
(\mathbf{n = 2 2})\end{array}$ & $\begin{array}{l}\text { Statistical } \\
\text { significance }\end{array}$ \\
\hline Age (Mean, SD) & 57.14, & 56.41, & 0.772 \\
& SD 8.96 & SD 8.73 & \\
Gender Male/Female & $21 / 8$ & $19 / 3$ & 0.230 \\
Education Less than 10 years/10 years or more & $13 / 16$ & $11 / 11$ & 0.382 \\
Marital Status Living/ Not living with a partner & $18 / 11$ & $17 / 5$ & 0.246 \\
Employment status Currently working/Not & $12 / 17$ & $13 / 9$ & 0.582 \\
working & & & \\
History of CHD & 9 & 8 & 0.69 \\
History of PCI & 1 & 0 & 0.60 \\
Revascularisation following ACS & 25 & 20 & 0.61
\end{tabular}


Table 2 Risk factor levels at baseline and 8-week follow-up

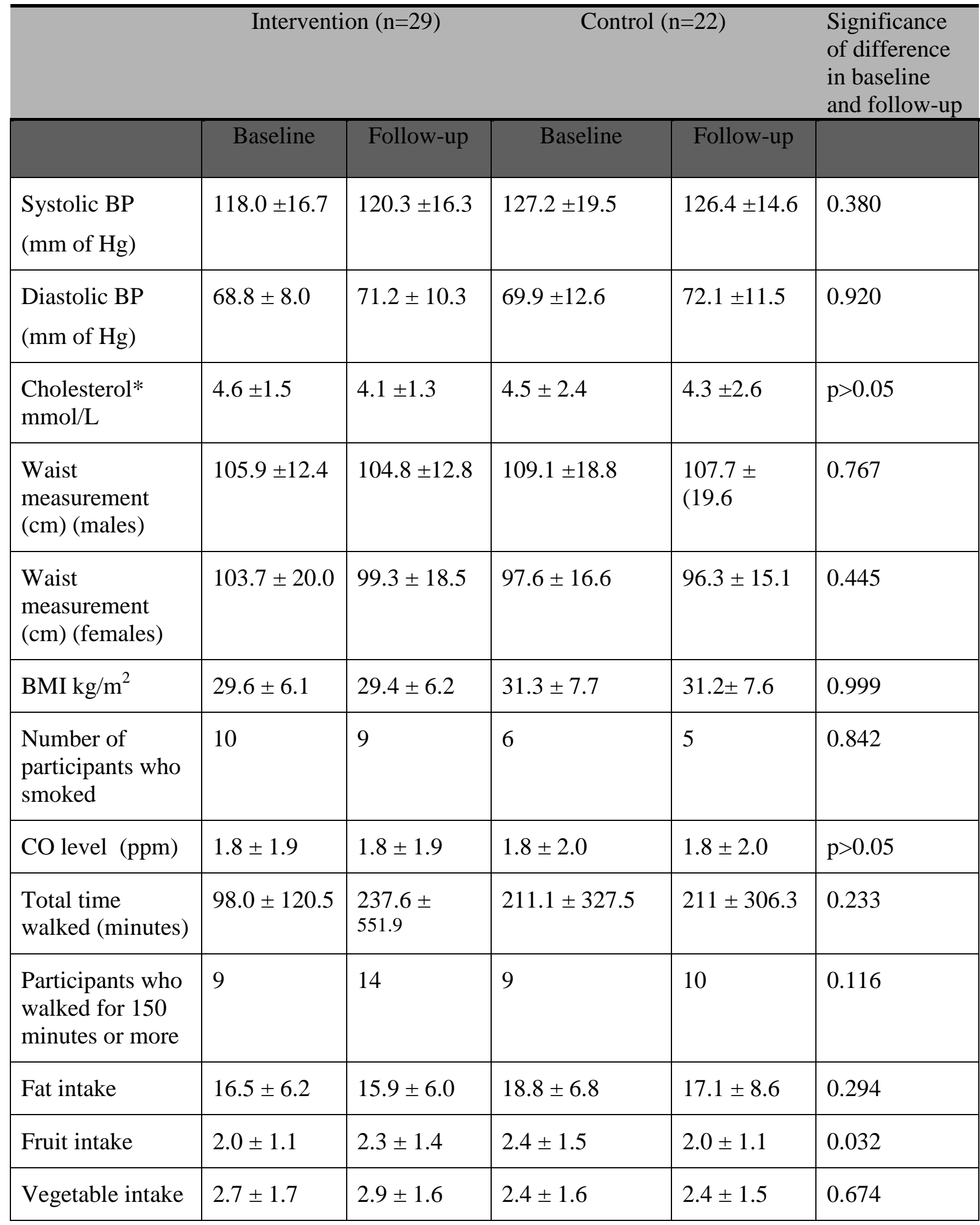

BP- blood pressure $\quad$ CO carbon monoxide $\quad$ BMI body mass index

* self report data missing 
Table 3 Satisfaction with the HeLM intervention (n=20)

Mean SD

Satisfaction with the HeLM booklet

Was the information provided was easy to read?

$8.8 \quad 0.97$

$\begin{array}{lll}\text { Was the information provided could be understood easily? } & 8.8 & 0.97\end{array}$

$\begin{array}{lll}\text { Was the information provided was useful? } & 8.7 & 1.0\end{array}$

After reading the booklet were you able to see the benefits of $8.9 \quad 0.8$

adopting healthy habits?

$\begin{array}{lll}\text { After reading the booklet were you able to increase your } & 8.8 & 1.09\end{array}$

confidence in being healthy?

$\begin{array}{lll}\text { After reading the booklet were you able to learn to cope with } \quad 8.2 & 1.48\end{array}$

difficult situations?

\section{Satisfaction with the risk factor card}

Did the risk factor card make you think about the health

8.0

1.49

related behaviour that you could change?

\section{Satisfaction with the telephone support}

Has the conversation with the nurse motivated you to make

8.2

0.78

healthy changes to your lifestyle?

Was the information given in the telephone call useful?

7.9

1.37 


\section{REFERENCES}

1. Marmot M, Elliott P. Coronary Heart Disease Epidemiology from aetiology to public health. Second ed. London: BMJ; 2005.

2. AIHW: Mathur S. Epidemic of coronary heart disease and its treatment in Australia. Canberra: Australian Institute of health and Welfare; 2002. Report No.: Cardiovascular disease Series No. 20 AIHW Cat. No. CVD 21.

3. Tod AM, Lacey EA, McNeill F. 'I'm still waiting...': barriers to accessing cardiac rehabilitation services. J Adv Nurs 2002; 40:421-431.

4. Yusuf S, Hawken S, Ounpuu S, Dans T, Avezum A, Lanas F, McQueen M, Budaj A, Pais P, Varigos J, Lisheng L, Investigators IS. Effect of potentially modifiable risk factors associated with myocardial infarction in 52 countries (the INTERHEART study): casecontrol study. Lancet 2004; 364:937-952.

5. Reimer W, de Swart E, De Bacquer D, Pyörälä K, Keil U, Heidrich J, Deckers J, Kotseva K, Wood D, Boersma E, EUROASPIRE Investigators. Smoking behaviour in European patients with established coronary heart disease. Eur Heart J 2006; 27:35-41.

6. National Heart Foundation of Australia, Cardiac Society of Australia and New Zealand. Reducing risk in heart disease 2007. A summary guide for preventing cardiovascular events in people with coronary artery disease (May 2004 2007).

7. Sundararajan V, Bunker SJ, Begg S, Marshall R, McBurney H. Attendance rates and outcomes of cardiac rehabilitation in Victoria, 1998. Med J Aust 2004; 180:268-271.

8. Vale MJ, Jelinek MV, Best JD, Santamaria JD. Coaching patients with coronary heart disease to achieve the target cholesterol: a method to bridge the gap between evidence-based medicine and the "real world"--randomized controlled trial. J Clin Epidemiol 2002; 55:245252.

9. Redfern J, Ellis E, Briffa T, Freedman SB. Modular prevention of heart disease following acute coronary syndrome (ACS). BMC Cardiovascular Disorders 2006; 6.

10. Hajek P, Taylor TZ, Mills P. Brief intervention during hospital admission to help patients to give up smoking after myocardial infarction and bypass surgery: randomised controlled trial. Br Med J 2002; 324:87-89. 
11. Daly J, Sindone AP, Thompson DR, Hancock K, Chang E, Davidson P. Barriers to participation in and adherence to cardiac rehabilitation programs: a critical literature review. Prog Cardiovasc Nurs 2002; 17:8-17.

12. Witt BJ, Thomas RJ, Roger VL. Cardiac rehabilitation after myocardial infarction: a review to understand barriers to participation and potential solutions. Europa Medicophysica 2005; 41:27-34.

13. Fernandez R, Davidson P, Griffiths R, Salamonson Y. Cardiac rehabilitation coordinators' perceptions of patient-related barriers to implementing cardiac evidence-based guidelines $J$ Cardiovasc Nurs 2008:in press.

14. Miller ET, Spilker J. Readiness to change and brief educational interventions: successful strategies to reduce stroke risk. J Neurosci Nurs 2003; 35:215-222.

15. Fernandez R, Griffiths R, Everett B, Andrew S, Salamonson Y, Davidson P. Effectiveness of brief structured interventions on risk factor modification for patients with coronary heart disease: A systematic review. Int J of Evidence-Based Health Care 2007.

16. Armstrong M. A Handbook of Human Resource Management Practice. 10th ed. London: Kogan Page; 2006.

17. Noar SM, Benac CN, Harris MS. Does tailoring matter? Meta-analytic review of tailored print health behavior change interventions. Psychol Bull 2007; 133:673-693.

18. Flesch R. A new readability yardstick. J Applied Psychol 1948; 32:221-233.

19. Fernandez RS, Griffiths R, Juergens C, Davidson P, Salamonson Y. Persistence of coronary risk factor status in participants 12 to 18 months after percutaneous coronary intervention. $J$ Cardiovasc Nurs 2006; 21:379-387.

20. Wilson PW, D'Agostino RB, Levy D, Belanger AM, Silbershatz H, Kannel WB. Prediction of coronary heart disease using risk factor categories. Circulation 1998; 97:1837-1847

21. National Heart Foundation of Australia, Cardiac Society of Australia and New Zealand. Management of unstable angina-Guidelines 2000. Med J Aust 2000; 173:S65-S88.

22. Edward T, Middleton B, Alyn H, ea. Breath carbon monoxide as an indication of smoking habit. Chest 2000; 117:758-763. 
23. O’Dea K, Walker K, Colagiuri S, Hepburn A, Holt P, Colagiuri R, 2002. Evidence Based Guidelines for Type 2 Diabetes Mellitus. Primary Prevention. Canberra: Diabetes Australia National Health and Medical Research Council (NHMRC); 2002.

24. Dobson AJ, Blijlevens R, Alexander HM, Croce N, Heller RF, Higginbotham N, Pike G, Plotnikoff R, Russell A, Walker R. Short fat questionnaire: a self-administered measure of fat-intake behaviour. Aust J Public Health 1993; 17:144-149.

25. Bland M. An Introduction to Medical Statistics. 3rd ed. Oxford: Oxford University Press; 2000.

26. National Heart Foundation of Australia, Cardiac Society of Australia and New Zealand. Reducing risk in heart disease 2004. A summary guide for preventing cardiovascular events in people with coronary artery disease (May 2004 2004).

27. Pickering TG, Hall JE, Appel LJ, Falkner BE, Graves JW, Hill MN, Jones DH, Kurtz T, Sheps SG, Roccella EJ, Council on High Blood Pressure Research P, Public Education Subcommittee AHA. Recommendations for blood pressure measurement in humans: an AHA scientific statement from the Council on High Blood Pressure Research Professional and Public Education Subcommittee. J of Clini Hyperten 2005; 7:102-109.

28. Fagerstrom K. Time to first cigarette; the best single indicator of tobacco dependence? Monaldi Arch Chest Dis 2003; 59:91-94.

29. Resnicow K, Campbell MK, Carr C, McCarty F, Wang T, Periasamy S, Rahotep S, Doyle C, Williams A, Stables G. Body and soul. A dietary intervention conducted through AfricanAmerican churches. Am J Prev Med 2004; 27:97-105.

30. New South Wales Health, Cancer Institute New South Wales. Go for 2 and 5 Sydney: New South Wales Health 2007.

31. Armstrong T, Bauman A, Davies J. Physical activity patterns of Australian adults. Results of the 1999 National Physical Activity Survey. Canberra: Australian Institute of Health and Welfare.; 2000.

32. Low EC, Ong MC, Tan M. Breath carbon monoxide as an indication of smoking habit in the military setting. Singapore Med J 2004; 45:578-582.

33. Middleton ET, Morice AH. Breath carbon monoxide as an indication of smoking habit. Chest 2000; 117:758-763. 
34. The New Zealand Guidelines Group (NZGG). New Zealand Cardiovascular disease risk calculator. http://www.nzgg.org.nz/guidelines/0035/CVD_Risk_Full.pdf\#page=26 (16th September 2005).

35. Aldana SG, Whitmer WR, Greenlaw R, Avins AL, Thomas D, Salberg A, Greenwell A, Lipsenthal L, Fellingham GW. Effect of Intense Lifestyle Modification and Cardiac Rehabilitation on Psychosocial Cardiovascular Disease Risk Factors and Quality of Life. Behav Modif 2006; 30:507-525.

36. Peterson ED, Lytle BL, Biswas MS, Coombs L. Willingness to participate in cardiac trials. Am J Geriatric Cardiol 2004; 13:11-15.

37. Berger VW, Weinstein S. Ensuring the comparability of comparison groups: is randomization enough? Control Clin Trials 2004; 25:515-524.

38. Rodgers A, Corbett T, Bramley D, Riddell T, Wills M, Lin RB, Jones M. Do u smoke after txt? Results of a randomised trial of smoking cessation using mobile phone text messaging. Tob Control 2005; 14:255-261. 